immunological methods alone (a group with a different prognosis).

Additionally, study criteria used to identify moderate to severe disease may be difficult to generalise. Subjective criteria such as "poor general condition" are difficult to assess and standardise in patients with malaria, even for specialist centres. Busy casualty departments in general hospitals will find it no easier. Even the harder criteria have pitfalls; in particular the admission of patients with a parasitaemia of $2 \%$ seems reassuring, but in the last 100 consecutive patients with falciparum malaria seen at our hospital, 23\% had an increase in parasitaemia over the first 24 hours of treatment, including eight increasing above $2 \%$, one with increase from $1.3 \%$ to $32 \%$, and one from $0.2 \%$ to $8.4 \%$. As a minor point, mefloquine, the main drug used in this study, is not used as first line treatment for malaria in most centres and may well be better adhered to by patients than quinine-which, although safe and effective, has major short term side effects and has to be taken for longer.

Conventional practice is to admit all patients with falciparum malaria because initial assessment can be misleading-even for specialist centres-and otherwise fit patients can deteriorate markedly, despite appropriate treatment. This study opens this practice up for debate, but it does not provide adequate justification for changing practice- - yet.

\title{
Hepatitis B immunisation in renal units in the United Kingdom: questionnaire study
}

\author{
Sunanda Ray, Terry Samuel, Jeremy Hawker, Steve Smith
}

Despite guidance from the Department of Health and the Renal Association that dialysis patients should be offered prophylaxis against hepatitis B by immunisation, surveys have shown that $95 \%$ of renal units in 1994 and 49\% in 1995 were not routinely offering immunisation to any patient groups with chronic renal failure. ${ }^{1-4}$ We aimed to determine whether provision of hepatitis B immunisation had improved after publication of the 1996 Department of Health guidelines and to identify barriers to implementation of existing guidelines. ${ }^{1}$

\section{Participants, methods, and results}

We sent a postal questionnaire, piloted in five renal units, to the clinical directors of all 87 main UK renal units and satellites. The questionnaire (available on bmj.com) covered hepatitis B immunisation in patients with chronic renal failure, including those receiving renal replacement therapy; the number of cases of acute hepatitis B infection between 1997 and 1999; and reasons why patients might not be vaccinated.

Seventy eight (90\%) units responded. Units in two teaching and four district general hospitals plus three satellites did not respond, despite reminders. Twelve units $(15 \%)$ reported at least one incident of hepatitis B seroconversion in a dialysis patient. Twenty three units $(29 \%)$ did not immunise any patient groups. A further six units offered immunisation only to patients planning treatment in hepatitis B endemic areas outside the United Kingdom.

Completeness of hepatitis B immunisation in dialysis patients was not known in 27 units $(35 \%)$, less than $25 \%$ in 17 units (22\%), $25-75 \%$ in 13 units (17\%), and over $75 \%$ in 20 units (26\%). Of the 55 units that provided immunisation, $70 \%$ gave the recommended higher dose of $40 \mu \mathrm{g}$ whereas $30 \%$ gave the previously recommended dose of $20 \mu \mathrm{g}$. Most (72\%) used the earlier schedule of doses at 0,1 , and 6 months instead of the recommended accelerated schedule of $0,1,2$, and 12 months. The table lists the reasons why patients are not routinely immunised.

Thirty six units (46\%) followed the Renal Association's recommendations on hepatitis B immunisation of patients with chronic renal failure; 42 did not. Fourteen units had developed their own policies. Eleven units (14\%) mentioned alternative guidance on immunisation, including the Department of Health's "green book" on infectious diseases, ${ }^{1}$ the revised Rosenheim report (the draft Department of Health's policy in development), ${ }^{4}$ and the British National Formulary.

One unit feared that staff might become less careful with universal precautions if all patients were immunised. Two units thought that the heavy workload produced little benefit. One unit abandoned an immunisation programme it had started after a seroconver-

\section{Communicable \\ Disease \\ Surveillance Centre, Birmingham Heartlands Hospital, Birmingham B9 5SS \\ Sunanda Ray specialist registrar in public health Jeremy Hawker regional epidemiologist \\ Renal Unit, Birmingham Heartlands Hospital Terry Samuel specialist registrar Steve Smith consultant \\ Correspondence to: Dr S Smith, Birmingham Heartlands Hospital, Bordesley Green East, Birmingham B9 5ST smiths@heartsol. wmids.nhs.uk}

BMJ 2002;324:877-8 Reasons given by 78 renal units as to why dialysis patients are not routinely vaccinated for hepatitis B before starting dialysis and during dialysis. Values are numbers (percentages) of units

\begin{tabular}{|c|c|c|}
\hline Reason & Before dialysis & During dialysis \\
\hline Logistics of administration and monitoring & $34(44)$ & $35(45)$ \\
\hline Low perceived risk (outbreaks rare) & $26(33)$ & $38(49)$ \\
\hline Awaiting revised guidelines from units committee & $24(31)$ & $26(33)$ \\
\hline Effectiveness of universal precautions and screening of blood donors and patients & $23(29)$ & $28(36)$ \\
\hline Poor efficacy of vaccine in patients receiving dialysis & $22(28)$ & $35(45)$ \\
\hline Should be done in primary care & $21(27)$ & $20(26)$ \\
\hline Not cost effective & $19(24)$ & $22(28)$ \\
\hline Lack of awareness of higher dose $(40 \mu \mathrm{g})$ vaccine & $14(18)$ & $16(21)$ \\
\hline
\end{tabular}


sion incident in 1996, owing to logistical difficulties. Two units mentioned difficulties in tracking patients who had been referred to general practice; one unit reverted to immunisation through dialysis services rather than primary care after seroconversion of a patient who had been referred. Six units mentioned costs and funding as barriers. One unit thought our survey would encourage provision of funding.

\section{Comment}

Although the rate of hepatitis B immunisation of patients with chronic renal failure in the United Kingdom has improved in recent years, most renal units still fail to follow current guidance. Partial coverage is the norm, and outmoded regimens are still used. The shared care management of immunisation may be one solution, although this requires good collaboration between primary and specialist care. Strategies that may improve collaborative care are inclusion of immunisation in service agreements, definition of responsibilities for initiation of immunisation, follow up and evaluation of response, payment to general practition- ers, and regular audit and shared feedback. The efficacy of the hepatitis B vaccine in end stage renal disease needs investigation to encourage its use in dialysis patients.

We thank all UK renal units who completed the study questionnaire and the staff at the Communicable Disease Surveillance Centre, Birmingham Heartlands Hospital.

Contributors: SS and JH had the original idea for this study SR designed the study questionnaire and played a key part in data collection, data documentation, and analysis. TS participated in the study design, data collection, and analysis. SR and TS jointly wrote the paper. SS and JH edited the paper. SS will act as guarantor for the paper.

Funding: None.

Competing interests: None declared.

1 Department of Health. Immunisation against infectious disease 1996. London: Stationery Office, 1996

2 Royal College of Physicians, Renal Association. Treatment of adult patients with renal failure. Recommended standards and audit measures. London: RCP, 1997:64-74

3 Jibani MM, Heptonstall J, Walker AM, Bloodworth LO, Howard AJ Hepatitis B immunization in UK renal units: failure to put policy into practice. Nephrol Dial Transplant 1994:9:1765-8.

4 Draft good practice guidelines for the prevention and control of blood borne virus infection in renal dialysis and renal transplantation units. London: Department of Health and Public Health Laboratory Service (in press). (Accepted 29 August 2001)

\section{Workplace bullying in junior doctors: questionnaire survey}

Lyn Quine

Centre for Research in Health

Behaviour, Department of

Psychology,

University of Kent

at Canterbury

Canterbury

CT2 7NP

Lyn Quine

reader in health

psychology

L.Quine@ukc.ac.uk

BMJ 2002;324:878-9
In the United Kingdom a growing literature has identified workplace bullying as a major occupational stressor among health professionals. A study carried out in an NHS community trust found that 1 in 3 staff reported being bullied in the previous year, ${ }^{1}$ while a report by the King's Fund, an independent health think tank, found that bullying, racial harassment, and discrimination were daily experiences for black and Asian doctors. In the United States several studies have reported that medical students suffer high levels of mistreatment or bullying during training, which increase with progression through medical school, spilling over into the early training years. ${ }^{2-4}$ We report here findings from a study of workplace bullying among junior doctors in the United Kingdom.

\section{Participants, methods, and results}

An anonymous questionnaire was sent out with $B M A$ News Review to 1000 doctors with job grades from house officer to senior registrar, randomly selected from the BMA members' mailing list. The questionnaire collected information about the participant's age, sex, job grade, and ethnic group. Participants were presented with a definition of bullying and asked to indicate whether they had been subjected to it in the past 12 months and whether they had witnessed others being bullied. They also completed a bullying scale which asked whether they had experienced 21 bullying behaviours from peers, senior staff, or managers in the past 12 months. ${ }^{1}$

The response rate was 62\%: 594 competed questionnaires were returned and 48 were returned undelivered by the post office. Not all questions were answered by all participants. Fifty four per cent (321) of the participants were house officers or senior house officers, 39\% (230) registrars, 3\% (18) senior registrars, and 3\% (20) other junior grades. Half were men (294v $296)$ and $70 \%$ (413 $v 174)$ were white. Overall, 220 of the 594 junior doctors (37\%) identified themselves as having been bullied in the past year, though $486(84 \%)$ had in fact experienced one or more of the bullying behaviours described on the bullying scale; 407 (69\%) had witnessed the bullying of others. Black and Asian doctors were more likely to report being bullied than white doctors $\left(78(45 \%)\right.$ v $139(34 \%) ; \chi^{2}=6.3, \mathrm{df}=1$, $\mathrm{n}=585, \mathrm{P}=0.01$; relative risk 1.59 (95\% confidence interval 1.11 to 2.28$)$ ) and women were more likely to report being bullied than men (43\% (126) v 32\% (92); $\chi^{2}=7.7, \mathrm{df}=1, \mathrm{n}=588, \mathrm{P}=0.005$; relative risk 1.61 (1.14 to 2.26); see table). Reports of bullying did not vary by job grade or age.

\section{Comment}

In this study $37 \%$ of junior doctors reported being bullied in the previous year and $84 \%$ had experienced at least one bullying behaviour. Black and Asian doctors were more likely to be bullied than other doctors. This should be a cause for concern, particularly since several recent studies show a pattern of discrimination at all levels in the medical profession from application to medical school to examination success, job application, and the allocation of distinction awards to consultants. ${ }^{5}$ Women were more likely than men to be bullied, and this finding is consistent with a study of university employees by Bjorkvist et al. ${ }^{4}$ 